\title{
The Use of Odd and Even Class Wind Speed Time Series of Distribution Histogram to Estimate Weibull Parameters
}

\author{
Adekunlé Akim Salami a,*, Ayité Sénah Akoda Ajavon a, Mawugno Koffi Kodjo a, \\ Seydou Ouedraogo ${ }^{b}$ and Koffi-Sa Bédja ${ }^{a}$
}

a Equipe de Recherche en Sciences de l'Ingénieur (ERSI), Department of Electrical Engineering, Ecole Nationale Supérieure d'Ingénieurs (ENSI), University of Lomé, BP 1515, Lomé, Togo

${ }^{b}$ Polytechnic University of bobo-Dioulasso, Burkina-Faso

\begin{abstract}
In this article, we introduced a new approach based on graphical method (GPM), maximum likelihood method (MLM), energy pattern factor method (EPFM), empirical method of Justus (EMJ), empirical method of Lysen (EML) and moment method (MOM) using the even or odd classes of wind speed series distribution histogram with $1 \mathrm{~m} / \mathrm{s}$ as bin size to estimate the Weibull parameters. This new approach is compared on the basis of the resulting mean wind speed and its standard deviation using seven reliable statistical indicators (RPE, RMSE, MAPE, MABE, $\mathrm{R}^{2}$, RRMSE and IA). The results indicate that this new approach is adequate to estimate Weibull parameters and can outperform GPM, MLM, EPF, EMJ, EML and MOM which uses all wind speed time series data collected for one period. The study has also found a linear relationship between the Weibull parameters K and C estimated by MLM, EPFM, EMJ, EML and MOM using odd or even class wind speed time series and those obtained by applying these methods to all class (both even and odd bins) wind speed time series. Another interesting feature of this approach is the data size reduction which eventually leads to a reduced processing time.
\end{abstract}

Keywords: Odd bin wind speed time series, Even bin wind speed time series, Weibull parameters, Statistical analysis, Comparative evaluation.

Article History: Received February 16 ${ }^{\text {th }}$ 2018; Received in revised form May $5^{\text {th }}$ 2018; Accepted May 27th 2018; Available online

How to Cite This Article: Salami, A.A., Ajavon, A.S.A., Kodjo, M.K., Ouedraogo, S. and Bédja, K. (2018) The Use of Odd and Even Class Wind Speed Time Series of Distribution Histogram to Estimate Weibull Parameters. Int. Journal of Renewable Energy Development 7(2), $139-150$. https://doi.org/10.14710/ijred.7.2.139-150

\section{Introduction}

In the last century, several climate changes have been observed in regions all over the world. The main cause of these climatic changes is the rise in fossil fuel use, which is due to the important demographic and industrial developments. These negative effects have forced scientists to draw attention to renewable energy sources such as solar, wind and hydraulic energy, which are the most suitable solution in the future (Dahmouni et al. 2011). Recently, interest in wind energy has been growing and many researchers have attempted to develop and to perform reliable wind energy conversion systems.

Wind energy conversion systems design required considerable efforts in recognizing a suitable statistical model for wind speed frequency distribution. The widely used function to model wind speed data is the Weibull distribution function(Celik, 2004). Recently it has become a reference distribution function in commercially used wind energy software i-e the Wind Atlas Analysis and Application Program (Sahin, 2004). We characterized the Weibull distribution by two parameters, a scale and a shape parameter (C and K) (Salami et al., 2016).
To estimate Weibull parameters several methods have been suggested in the literature, such as the graphical method (GPM), the maximum likelihood method (MLM), the moment method (MOM), the empirical method of Justus (EMJ), the empirical method of Lysen (EML), the method of the modified maximum likelihood (MMLM), the equivalent energy method (EQM)(Rocha et al., 2012) and the energy pattern factor method (EPFM) (Kasra et al., 2016).

To estimate Weibull parameters for a site using the methods frequently used and cited above, the wind speed time series data collected in an interval of one (1) hour in a given period are used (Garcia et al., 1998). The recent works of (Yuan et al., 2015) aims to compare the performance of the MLM and the MOM. The results show that for an extreme small data size, the MLM slightly outperforms the MOM and generally, the MLM has an advantage over medium and large data sizes. In conclusion, for life data analysis, it is suggested to use MLM for the two-parameter Weibull distribution. Given that, wind speed distribution is used in estimating Weilbull parameters; we argue that it is possible to reduce the length of the input series through filtering. A reduced data size will lead to higher computational efficiency in estimating Weibull parameters and key

\footnotetext{
* Corresponding author: akim_salami@yahoo.fr
} 
wind site characteristics such us mean wind speed and wind speed standard deviation. The main question to address is what filtering to perform in order to achieve this much-desired computational efficiency while maintaining acceptable estimation accuracy? To the best of our knowledge, no existing work proposed a filtering method that allies accuracy and efficiency for Weibull parameter estimation in wind energy applications. This paper proposes the following contributions.

In order to reduce data size and therefore parameter estimation time, while maintaining a high accuracy of mean wind speed and standard deviation, a new approach is proposed. Indeed, the series of wind speed is grouped in classes (or bins), each class being represented by a bin in the distribution histogram. The set of classes is divided into two subsets: even and odd order speed classes. In this paper, we aim to adequately determine the overall Weibull parameters ( $\mathrm{K}$ and $\mathrm{C}$ ) using those estimated for the subsets of odd and even speed classes. For each subset, the Weibull parameters are estimated using six well known methods namely graphical method (GPM), empirical method of Justus (EMJ), empirical method of Lysen (EML), energy pattern factor method (EPFM), maximum likelihood method (MLM) and moment method (MOM). So in this article eighteen methods are used to estimate Weibull parameters for a given period at a geographic locality, these are:

- GPM, EMJ, EML, EPFM, MLM, MOM using all bins wind speed time series;

- GPM, EMJ, EML, EPFM, MLM, MOM using Odd Bins wind speed time series (GPOBM, EMJOB, EMLOB, EPFOBM, MLOBM, MOOBM);

- GPM, EMJ, EML, EPFM, MLM, MOM using Even Bins wind speed time series (GPEBM, EMJEB, EMLEB, EPFEBM, MLEBM, MOEBM).

Consequently, in this study the capability of eighteen parameter estimation methods is evaluated to calculate the $\mathrm{K}$ and $\mathrm{C}$ parameters for adjusting the Weibull distribution of wind speeds. The main goal is identifying the most appropriate method for computing the mean and standard deviation at two sites (Lomé in Togo and Ouagadougou in Burkina Faso). To achieve this, a comprehensive statistical analysis based upon several statistical parameters and approaches is conducted using the eighteen parameter estimation methods.

The rest of this paper is structured as follows. Section 2 describes numerical methods for determining the Weibull parameters. Section 3 presents in detail the proposed approaches to estimate Weibull parameters. In Section 4, statistical indicators for performance evaluation are illustrated. The results and discussions along with the underlying case study data are presented in Section 5. Finally, conclusions are drawn in Section 6.

\section{Numerical Methods for determining the Weibull parameters}

Wind speed is a random variable, and to determine the wind potential of a region it is necessary to use statistical analysis (Salami et al., 2013). This requires the existence of time series records of wind speed.

Such records are the wind data. Based on the wind speed data collected, the Weibull distribution can be described as a probability density function $f(V)$ and a cumulative distribution function $\mathrm{F}(\mathrm{V})$ are respectively determined by Eqs. (1) and (2) (Ahmed, 2013):

$$
\begin{aligned}
& f(V)=\left(\frac{K}{C}\right)\left(\frac{V}{C}\right)^{K-1} \exp \left[-\left(\frac{V}{C}\right)^{K}\right] \\
& F(V)=\left(1-\exp \left[-\left(\frac{V}{C}\right)^{K}\right]\right)
\end{aligned}
$$

The mean and standard deviation of the wind speed series are given by Eq. (3). and Eq. (4).

$$
\begin{aligned}
& \bar{V}=\frac{1}{n} \sum_{i=1}^{n} V_{i}=C \Gamma\left(1+\frac{1}{K}\right) \\
& \sigma=\left[\frac{1}{n-1} \sum_{i=1}^{n}\left(V_{i}-\bar{V}\right)\right]^{1 / 2}=C\left[\Gamma\left(1+\frac{2}{K}\right)-\Gamma^{2}\left(1+\frac{1}{K}\right)\right]^{1 / 2}
\end{aligned}
$$

where: $\mathrm{C}$ and $\mathrm{K}$ are respectively scale and shape parameters of the Weibull distribution function and $\Gamma$ represents the gamma function defined by the Euler integral of the second kind.

Wind power density is an important indicator for determining the potential of wind resources and to describe the amount of wind energy at various wind speeds at a particular location. Knowledge of wind power density is also useful to evaluate the performance of wind turbines and select the optimum wind turbines. Wind power density resembles the level of accessible energy at the site which can be converted to electricity by wind turbines. Indeed, the mean kinetic energy, available at a site per unit of time and per unit area is expressed by Eq. (5) (Seguro et al., 2000):

$P=\frac{1}{2} \rho \int_{0}^{+\infty} V^{3} f(V) d V=\frac{1}{2} \rho \overline{V^{3}}=\frac{1}{2} \rho C^{3} \Gamma\left(1+\frac{3}{K}\right)$

where:

- $\quad \rho$ is air density $\left(\mathrm{kg} \mathrm{m}^{-3}\right)$,

- V is wind speed

- $f(V)$ is the Weibull probability distribution function (pdf) (Eq. 1),

- $\overline{\mathrm{V}^{3}}$ is the cubic mean wind speed.

There are some methods introduced in the literature (Dinler et al., 2009) to calculate the $\mathrm{K}$ and $\mathrm{C}$ parameters of the Weibull distribution function. In this study, six methods: graphical method (GPM), empirical method of Justus (EMJ), empirical method of Lysen (EML), energy pattern factor method (EPFM), maximum likelihood method (MLM) and moment method (MOM) frequently used to compute the $\mathrm{K}$ and $\mathrm{C}$ parameters are selected for comparative evaluation. The descriptions of these six methods are provided briefly in what follows.

\subsection{Graphical method (GPM)}

The graphical method is achieved through the cumulative distribution function. In this distribution method, wind speed data are interpolated by a straight line, using the concept of least squares. The equation for 
this method can be represented by a double logarithmic transformation (Rocha et al., 2012) as follows,

$\ln \{-\ln [1-F(V)]\}=K \ln (V)-K \ln (C)$

\subsection{Empirical method of Justus (EMJ)}

Based on the empirical method introduced by Justus, the $\mathrm{K}$ and $\mathrm{C}$ parameters are computed, respectively by Eq. (7) and Eq. (8)(Kasra et al., 2016):

$$
\begin{aligned}
& K=\left(\frac{\sigma}{\bar{V}}\right)^{-1.086} \\
& C=\frac{\bar{V}}{\Gamma\left(1+\frac{1}{K}\right)}
\end{aligned}
$$

where:

- $\overline{\mathrm{V}}$ is the mean wind speed,

- $\sigma$ is the standard deviation of the observed data,

- $\quad \Gamma(x)$ is the gamma function.

\subsection{Empirical method of Lysen (EML)}

In the empirical method suggested by Lysen, $\mathrm{K}$ is calculated by Eq. (7) same as the Justus method. In fact, the only difference is the equation of $\mathrm{C}$. In the empirical method of Lysen, C is obtained by Eq. (9) (Kasra et al., 2016):

$$
C=\bar{V}\left(0.568+\frac{0.433}{K}\right)^{-\frac{1}{K}}
$$

\subsection{Energy pattern factor method (EPFM)}

The energy pattern factor method is related to the averaged data of wind speed and is defined by the following equations (Kasra et al., 2016):

$$
\begin{aligned}
& E_{p f}=\left(\frac{\overline{V^{3}}}{\bar{V}}\right) \\
& K=\left(1+\frac{3.69}{E_{p f}^{2}}\right)
\end{aligned}
$$

and parameter $\mathrm{C}$ is also computed similarly as in the empirical method of Justus by Equation (8).

\subsection{Maximum likelihood method (MLM)}

The maximum likelihood estimation method is difficult to solve, since numerical iterations are needed to determine the parameters of the Weibull distribution (Kidmo et al. 2015). In this method, the parameters $\mathrm{K}$ and $\mathrm{C}$ are determined according to the equations below:

$$
\begin{aligned}
& K=\left[\left[\frac{\sum_{i=1}^{n} V_{i}^{K} \ln \left(V_{i}\right)}{\sum_{i=1}^{n} V_{i}^{K}}\right]-\left[\frac{\sum_{i=1}^{n} \ln \left(V_{i}\right)}{n}\right]\right]^{-1} \\
& C=\left[\frac{\sum_{i=1}^{n} V_{i}^{K}}{n}\right]^{1 / K}
\end{aligned}
$$

\subsection{Moment method (MOM)}

The moment method can be used as an alternative to the maximum likelihood method and, in this case, the parameters $\mathrm{K}$ and $\mathrm{C}$ are respectively determined by Eq. (14) and Eq. (15) (Mostafaeipour et al. 2011).

$$
\begin{aligned}
& \bar{V}=C \Gamma\left(1+\frac{1}{K}\right) \\
& \sigma=C\left[\Gamma\left(1+\frac{2}{K}\right)-\Gamma^{2}\left(1+\frac{1}{K}\right)\right]^{1 / 2}
\end{aligned}
$$

\section{Proposed approaches to Weibull parameter estimation}

The information contained in the wind measurements at a given site can be represented as a histogram. Given $\left(\mathrm{V}_{1}, \mathrm{~V}_{2}, \mathrm{~V}_{3}, \quad, \mathrm{~V}_{\mathrm{r}}\right)$, $\mathrm{r}$ wind speeds measured at a site, this sequence can be grouped into $\mathrm{m}(\mathrm{m} \leq \mathrm{r})$ classes $\left(\operatorname{Bin}_{0}, \operatorname{Bin}_{1}, \operatorname{Bin}_{2}, \quad, \operatorname{Bin}_{m-1}\right) ;$ let $f_{j}$, be the relative frequency of class $\operatorname{Bin}_{j}$, the graph $\left(\operatorname{Bin}_{j} \times f_{j}\right.$, $)$ represents the histogram of the distribution of relative frequencies of wind speed at this site.

Thus, for all $\mathrm{n}$ samples of wind measurements $\left(\mathrm{V}_{1}, \mathrm{~V}_{2}, \mathrm{~V}_{3}, \quad \mathrm{~V}_{\mathrm{n}}\right)$ obtained during a period of time at a given site, the application of the 6 selected methods (GPM, MLM, EPFM, EMJ, EML and MOM) gives the shape $\mathrm{K}$ and scale $\mathrm{C}$ parameters of the Weibull.

In order to reduce the number of data to be processed while maintaining accurate standard deviation and mean wind speed, all samples of $\mathrm{n}$ wind measurements $\left(\mathrm{V}_{1}, \mathrm{~V}_{2}, \mathrm{~V}_{3}, \quad \mathrm{~V}_{\mathrm{n}}\right)$ obtained during a period of time at a given site are grouped into classes and represented as a histogram (the graph $\left(\operatorname{Bin}_{\mathrm{j}} \times \mathrm{f}_{\mathrm{j}}\right)$ ). The obtained wind speed classes can be divided into two groups: the group of even speed classes $\left(\operatorname{Bin}_{2 \mathrm{k}}\right)$ and the group of odd classes $\left(\operatorname{Bin}_{2 \mathrm{k}+1}\right)$.

Samples of $p$ wind speed measurements $\left(X_{1}, X_{2}, X_{3}, \quad, X_{p}\right)$ of the group of even classes group $\left(\operatorname{Bin}_{2 \mathrm{k}}\right)$, subsets of $\left(\mathrm{V}_{1}, \mathrm{~V}_{2}, \mathrm{~V}_{3}, \mathrm{~V}_{\mathrm{n}}\right)$, are used to estimate the shape parameter $\mathrm{K}$ and scale parameter $\mathrm{C}$ using 6 selected methods. So this new approach is referred to as:

- graphical with Even Bin time series method (GPEBM) when GPM is used; 
- $\quad$ maximum likelihood with Even Bin time series method (MLEBM) when MLM is used;

- $\quad$ energy pattern factor with Even Bin time series method (EPFEBM) when EPFM is used;

- empirical method of Justus with Even Bin time series (EMJEB) when EMJ is used;

- empirical method of Lysen with Even Bin time series (EMLEB) when EML is used;

- moment with Even Bin time series method (MOEBM) when MOM is used.

Likewise, samples of $\mathrm{q}$ wind measurements $\left(\mathrm{Y}_{1}, \mathrm{Y}_{2}, \mathrm{Y}_{3}, \quad \mathrm{Y}_{\mathrm{q}}\right)$ of the group of odd classes $\left(\operatorname{Bin}_{2 \mathrm{k}+1}\right)$, subsets of $\left(V_{1}, V_{2}, V_{3}, \quad, V_{n}\right)$, are used to estimate $\mathrm{K}$ and $\mathrm{C}$ (shape and scale parameters) using 6 selected methods. So this new approach is referred to as:

- graphical with Odd Bin time series method (GPOBM) when GPM is used;

- maximum likelihood with Odd Bin time series method (MLOBM) when MLM is used;

- $\quad$ energy pattern factor with Odd Bin time series method (EPFOBM) when EPFM is used;

- empirical method of Justus Odd Bin time series (EMJOB) when EMJ is used;

- $\quad$ empirical method of Lysen Odd Bin time series (EMLOB) when EML is used;

- moment with Odd Bin time series method (MOOBM) when MOM is used.

Thus, this study aims to verify if, from each speed class group (even or odd) taken individually; it is possible to estimate the parameters ( $\mathrm{K}$ and $\mathrm{C}$ ) suitable for an accurate estimation of the mean wind speed and standard deviation at the Lomé and Ouagadougou sites.

\section{Statistical indicators used for performance evaluation}

To assess the performance of the eighteen parameter estimation methods of the Weibull distribution for estimating mean wind speed and standard deviation, different statistical approaches including seven reliable statistical indicators (Legates et al., 1999) have been used in this study. Several statistical parameters including relative percentage error (RPE), mean absolute percentage error (MAPE), mean absolute bias error (MABE), root mean square error (RMSE), relative root mean square error (RRMSE), correlation coefficient $\left(\mathrm{R}^{2}\right)$ and index of agreement (IA) (Kasra et al., 2016) along with some other statistical tools have been utilized to offer an appropriate comparative assessment. In the following, a brief description of the considered statistical parameters is offered

\subsection{Relative percentage error (RPE)}

The RPE shows the percentage deviation between the calculated wind speed and standard deviation from the Weibull function $\mathrm{xi}_{\mathrm{i}}$ and those obtained by measured values $\mathrm{y}_{i}$ and its values ranging between $-10 \%$ and $+10 \%$ which are usually considered acceptable. RPE is defined as:

$\operatorname{RPE}(\%)=100 \times\left(\frac{y_{i}-x_{i}}{y_{i}}\right)$ where:

- $\mathrm{N}$ is the number of observations,

- $\mathrm{y}_{\mathrm{i}}$ is the measured values,

- $\mathrm{X}_{\mathrm{i}}$ is the predicted values.

\subsection{Mean absolute percentage error (MAPE)}

The MAPE shows the mean absolute percentage difference between the computed mean wind speed and standard deviation using the Weibull function and those attained by measured values. The MAPE is calculated by Eq. (17):

$M A P E=\frac{1}{N} \sum_{i=1}^{N}\left|\frac{y_{i}-x_{i}}{y_{i}}\right| \times 100$

\subsection{Mean absolute bias error (MABE)}

The MABE represents the average quantity of total absolute bias errors between the calculated mean wind speed and standard deviation by Weibull function and those obtained by measured values. The MABE is defined by Eq. (18):

$M A B E=\frac{1}{N} \sum_{i=1}^{N}\left|y_{i}-x_{i}\right|$

\subsection{Root mean square error (RMSE)}

The RMSE identifies model accuracy by comparing the deviation between the values achieved by the Weibull function and those of measured data. The RMSE has always a positive value and it is calculated using Eq. (19):

$$
R M S E=\sqrt{\frac{1}{N} \sum_{i=1}^{N}\left(y_{i}-x_{i}\right)^{2}}
$$

\subsection{Relative root means square error (RRMSE)}

The RRMSE is obtained by dividing the RMSE by the average of mean wind speed and standard deviation obtained by measured values as follows:

$$
\operatorname{RRMSE}(\%)=\frac{\sqrt{\frac{1}{N} \sum_{i=1}^{N}\left(y_{i}-x_{i}\right)^{2}}}{\frac{1}{N} \sum_{i=1}^{N} y_{i}} \times 100
$$

Different ranges of RRMSE can be defined to represent the precision of the model as (Legates et al., 1999):

- Excellent for RRMSE $<10 \%$;

- Good for $10 \%<$ RRMSE $<20 \%$;

- $\quad$ Fair for $20 \%<$ RRMSE $<30 \%$;

- $\quad$ Poor for RRMSE $>30 \%$.

\subsection{Correlation coefficient $\left(R^{2}\right)$}

The $\mathrm{R}^{2}$ which indicates the strength of the linear relationship between the calculated mean wind speed 
and standard deviation by the Weibull function and those computed using measured values is calculated by Eq. (21):

$$
\mathrm{R}^{2}=\frac{\sum_{i=1}^{N}\left(x_{i}-\overline{x_{i}}\right)\left(y_{i}-\overline{y_{i}}\right)}{\sqrt{\sum_{i=1}^{N}\left(x_{i}-\overline{x_{i}}\right)^{2} \sum_{i=1}^{N}\left(y_{i}-\overline{y_{i}}\right)^{2}}}
$$

\subsection{Index of agreement (IA)}

The IA generally shows the degree of precision of the predicted values compared to the measured values. The IA which varies from 0 to 1 is calculated by Eq. (22):

$$
L A=\frac{\sum_{i=1}^{N}\left|y_{i}-x_{i}\right|}{\sum_{i=1}^{N}\left(\left|x_{i}-\overline{x_{i}}\right|+\left|y_{i}-\overline{y_{i}}\right|\right)}
$$

\section{Results and discussion}

\subsection{Case study and wind speed data}

The data for the two sites, Lomé in Togo and Ouagadougou in Burkina Faso were chosen for this study because several wind energy projects have become a necessity in both countries.

The demand for electricity in Togo continues to increase at an average rate of $8 \%$ per year and could double in the next 10 years. The country's electricity supply is heavily dependent on oil and its derivative products (including natural gas), which are subject to international price volatility (Kimatu et al., 2011). Togo is ranked among the quiet areas although transient spikes wind speed can reach high values up to $4 \mathrm{~m} / \mathrm{s}$ in some areas especially in the northern part of the country during the harmattan period. Only the coastal area of the country has favorable evidence with wind speeds of $3 \mathrm{~m} / \mathrm{s}$ on average. The development of wind power can be considered as a viable alternative. A project of two wind farms of $12 \mathrm{MW}$ each located near Lomé is undergoing by a private company named Eco Delta Development (EDD) and its subsidiary Delta Wind Togo.

In Burkina Faso, renewable energy concerns mainly the use of the wood fuel. Indeed, at household level in peri-urban and rural areas, biomass is the primary energy source for cooking. Due to accelerating urbanization in recent decades, services in peri-urban areas are collapsing under the strain of rapid development, resulting in uncontrolled sanitation problems and household energy insecurity. However, the further development of renewable energy technologies is only marginally supported by the government, despite its significant potential (Al-Mulali et al., 2012). Due to the western location of Burkina Faso, the potential for wind power is very limited. The average wind speed ranges between 1 and $3 \mathrm{~m} / \mathrm{s}$, with the maximum only obtained in the North. However, small-scale generators at suitable sites for selective purposes (e.g. water pumping, desalination systems etc.) might be feasible(Bugaje, 2006). Hourly mean wind speed data used for Lomé and Ouagadougou were obtained from the meteorological database at

http:/ / weather.uwyo.edu/area/meteorogram /'.

The coordinates of the two sites in our case study are given in Table 1 . The data is recorded every day at one hour intervals (this is the mean over the 10 minutes before the hour) at a height of $10 \mathrm{~m}$ above the ground.

Table 1

Coordinates of the case study sites

\begin{tabular}{lc}
\hline Sites & Coordinates \\
\hline Lomé & $6.17 \mathrm{~N}, 1.25 \mathrm{E}, 25$ meters \\
Ouagadougou & $12.35 \mathrm{~N}, 1.52^{\circ} \mathrm{W}, 306$ meters \\
\hline
\end{tabular}

Data collected cover the period, from January 2004 to December 2015 for the Lomé site (record length of approximately twelve (12) years) and from January 2009 to December 2015 for the Ouagadougou site (record length of approximately seven (7) years).

Using the methodology described earlier, we processed the 10-minute averaged hourly wind speed data collected at the Lomé and Ouagadougou sites. In order to assess the performance of our proposed approach, it is important to apply it over several periods. Given the importance of the analysis of monthly variations of wind characteristics at a given site, our case study covers each month (the entire dataset is grouped monthly into 12 study periods: January, February, March, April, May, June, July, August, September, October, November, December), each month of each year and the aggregate for the whole year. As the data collected at the Lomé site covers a period from January 2004 to December 2015, the total number of periods of the study is 157 for the Lomé site. Total study periods considered for the Ouagadougou site is 97 , since the data collected on this site covers a period from January 2009 to December 2015. For each given period and each of the two sites considered, we classified wind speeds in bins of size $1 \mathrm{~m} / \mathrm{s}$ each according to Table 2.

For each period and each site, the results are presented and analyzed. Table 3 and 4 present some descriptive statistics including maximum, mean, standard deviation, skewness and kurtosis of the used wind speed data at the two sites for even, odd and all classes of wind speed data subset.

Table 2

Wind speed classes adopted for the two sites.

\begin{tabular}{lcr}
\hline Wind speed $(\mathbf{m} / \mathbf{s})$ & Bins & Type \\
\hline$] 0,1[$ & $\operatorname{Bin}_{0}$ & Even bin \\
{$[1,2[$} & $\operatorname{Bin}_{1}$ & Odd bin \\
{$[2,3[$} & $\operatorname{Bin}_{2}$ & Even bin \\
$\ldots$ & $\ldots$ & $\ldots$ \\
$\ldots$ & $\ldots$ & $\ldots$ \\
] $21,22[$ & $\ldots$ & Odd bin \\
\hline
\end{tabular}


Citation: Salami, A.A., Ajavon, A.S.A., Kodjo, M.K., Ouedraogo, S. and Bédja, K. (2018), The Use of Odd and Even Class Wind Speed Time Series of Distribution Histogram to Estimate Weibull Parameters. Int. Journal of Renewable Energy Development, 7(2), 139-150, doi.org/10.14710/ijred.7.2.139-150

P a g e 144

Table 3

Descriptive statistics of the used wind speed data according to wind speed classes for the Lomé site 2004-2015.

\begin{tabular}{lllllll}
\hline $\begin{array}{l}\text { Wind speed } \\
\text { classes (Bins) }\end{array}$ & $\begin{array}{l}\text { Rel. Freq. } \\
(\%)\end{array}$ & $\begin{array}{l}\text { Max. } \\
(\mathbf{m} / \mathbf{s})\end{array}$ & $\begin{array}{l}\text { Mean } \\
(\mathbf{m} / \mathbf{s})\end{array}$ & $\begin{array}{l}\text { Std. Dev. } \\
(\mathbf{m} / \mathbf{s})\end{array}$ & Kurt. & Skew. \\
\hline All & 100 & 16 & 3.52870 & 2.02964 & 2.33358 & 0.26247 \\
Odd & 51.46085 & 13 & 3.52034 & 2.03017 & 2.19198 & 0.32246 \\
Even & 48.53915 & 16 & 3.53756 & 2.02906 & 2.48615 & 0.19880 \\
\hline
\end{tabular}

Table 4

Descriptive statistics of the used wind speed data according to wind speed classes for the Ouagadougou site 2009-2015

\begin{tabular}{lllllll}
\hline $\begin{array}{l}\text { Wind speed } \\
\text { classes (Bins) }\end{array}$ & Rel. Freq. (\%) & $\begin{array}{l}\text { Max. } \\
(\mathbf{m} / \mathbf{s})\end{array}$ & $\begin{array}{l}\text { Mean } \\
(\mathbf{m} / \mathbf{s})\end{array}$ & $\begin{array}{l}\text { Std. Dev. } \\
(\mathbf{m} / \mathbf{s})\end{array}$ & Kurt. & Skew. \\
\hline All & 100 & 22 & 2.99562 & 1.66267 & 4.59290 & 0.78947 \\
Odd & 49.59290 & 15 & 3.06272 & 1.63272 & 4.40914 & 0.79136 \\
Even & 50.40710 & 22 & 2.92961 & 1.68904 & 4.76355 & 0.79843 \\
\hline
\end{tabular}

The mean wind speed and standard deviation are similar for all three data classes considered for each site. This is a preliminary indication that the Weibull parameters estimated from either even or odd class speed data subset might yield similar mean wind speed and standard deviation as the entire dataset (all class data).

As noticed, Lomé has the highest mean wind speed of $3.5287 \mathrm{~m} / \mathrm{s}$ and Ouagadougou has the lowest wind speed of $2.99562 \mathrm{~m} / \mathrm{s}$. Moreover, for the Ouagadougou site, the coefficient of Kurtosis is significantly higher than the Lomé site.

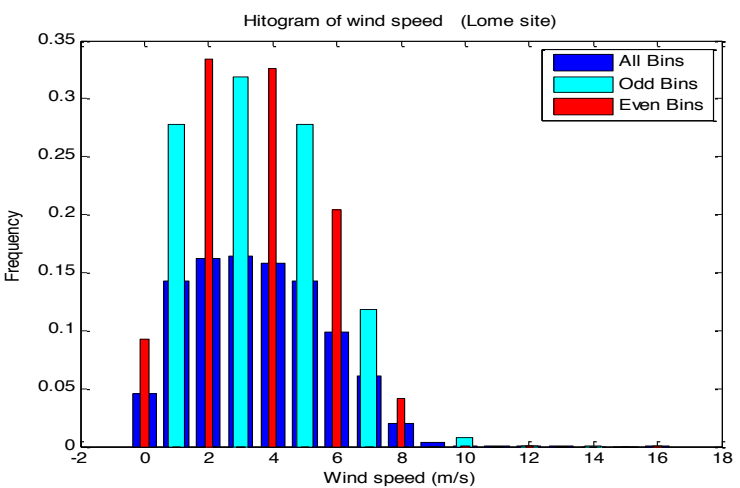

Fig. 1. Histograms of wind speed data according to the wind speed classes at the Lomé site.

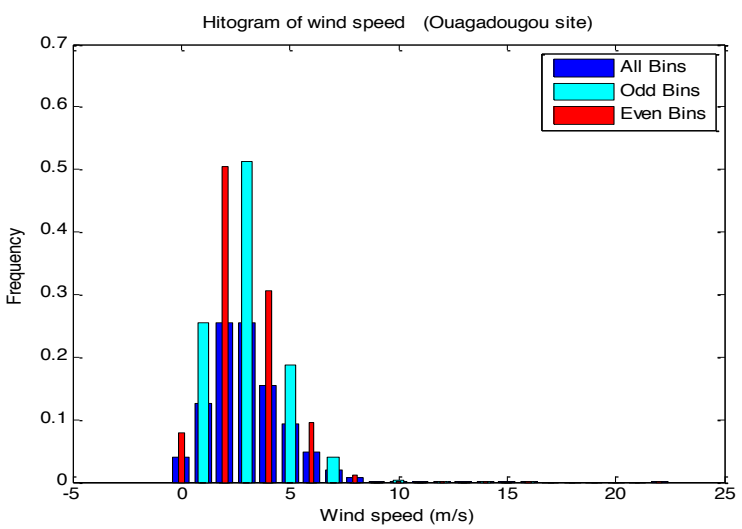

Fig. 2 Histograms of wind speed data according to the wind speed classes at the Ouagadougou site.
Fig. 1 and Fig. 2 show the probability densities of the utilized wind speed data according to the wind speed classes for two selected sites, respectively.

Also in order to show that the Weibull parameters can be estimated from even or odd class wind speed data for the periods considered at a site, we checked if there is a linear correlation between all class wind speed data characteristics (means and standard deviations of wind speed time series for chosen periods) calculated, and even or odd class wind speed data characteristics (means and standard deviations of wind speed time series for chosen periods) calculated.

The results in Fig. 3 and Fig. 4, show that there is:

- a strong linear correlation $\left(\mathrm{R}^{2}=0.95735\right)$ between the means of wind speed time series calculated considering all class data and the means of wind speed time series calculated considering the even class data first, against a linear correlation $\left(\mathrm{R}^{2}=\right.$ 0.80231) considering odd class data when the data collected at the Lomé site are used;

- a strong linear correlation $\left(\mathrm{R}^{2}=0.98234\right)$ between the means of wind speed time series calculated considering all class data and the means of wind speed time series calculated considering the even class data first, against a linear correlation $\left(\mathrm{R}^{2}=\right.$ 0.9705) considering odd class data when the data collected at the Ouagadougou site are used.

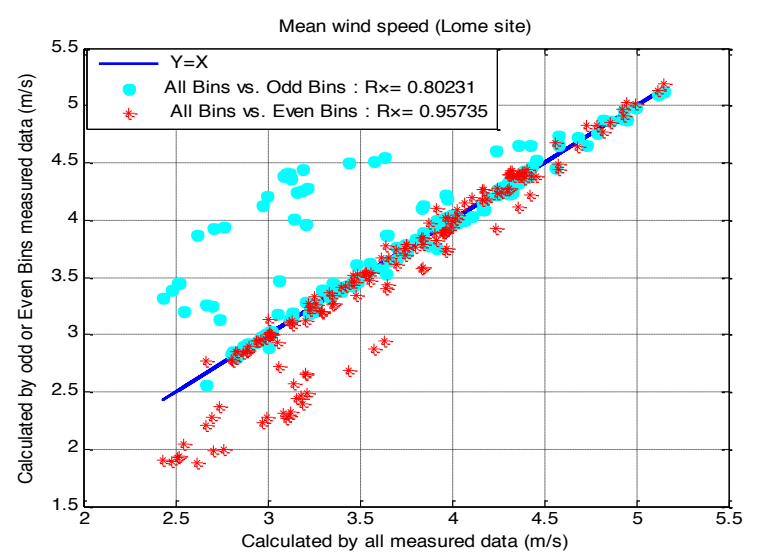

Fig. 3 Scatter plots of computed mean wind speed values using all bin time series versus those computed using even bin or odd bin wind speed time series for Lomé. 


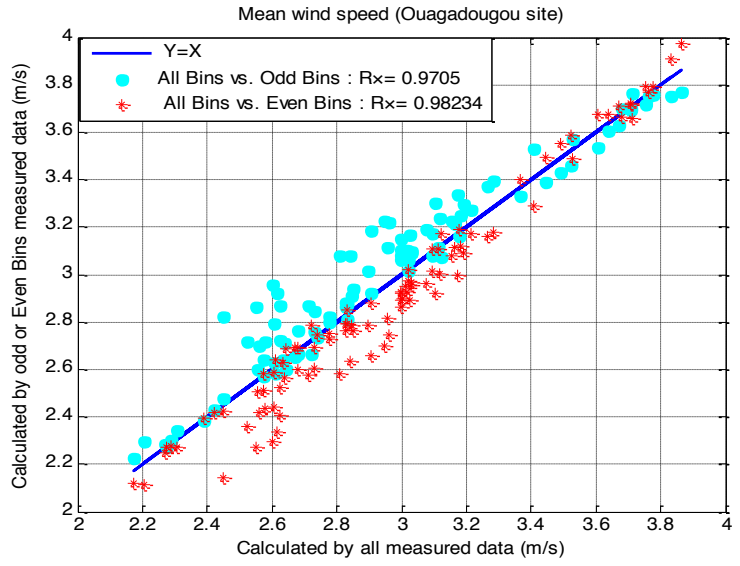

Fig. 4 Scatter plots of computed mean wind speed values using all bin time series versus those computed using even bin or odd bin wind speed time series for Ouagadougou.

From the results of Fig. 5 and Fig. 6, we note that there is:

- a strong linear correlation $\left(\mathrm{R}^{2}=0.95469\right)$ between the standard deviations of wind speed time series calculated considering all class data and standard deviations of wind speed time series calculated considering the even class data first, against low linear correlation $\left(\mathrm{R}^{2}=0.23878\right)$ considering odd class data when the data collected at the Lomé site are used;

- a strong linear correlation $\left(\mathrm{R}^{2}=0.96961\right)$ between the standard deviations of wind speed time series calculated considering all class data and standard deviations of wind speed time series calculated considering the even class data first, against a linear correlation $\left(\mathrm{R}^{2}=0.93225\right)$ considering odd class data when the data collected at the Ouagadougou site are used.

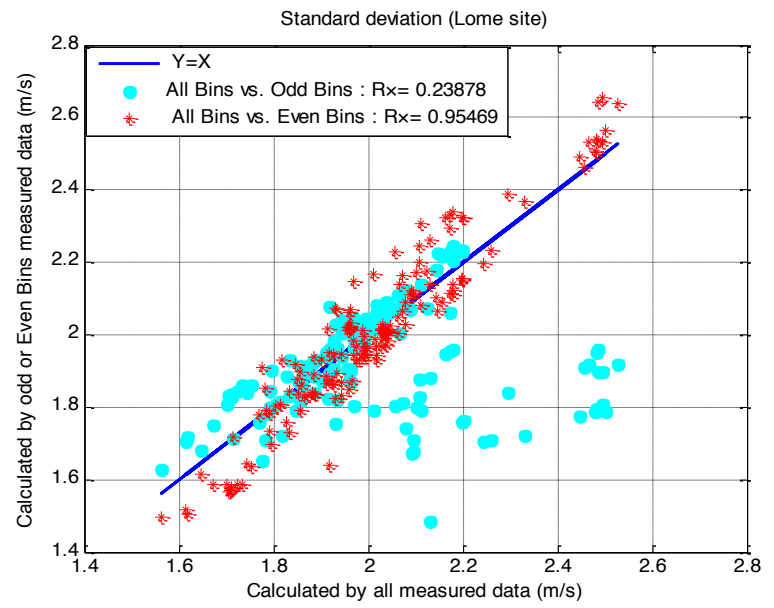

Fig. 5 Scatter plots of computed wind speed standard deviation values using all bin time series versus those computed using even bin or odd bin wind speed time series for Lomé.

From the results of Figs. 3, 4, 5 and 6, we can hope that we can estimate the Weibull parameters at two (02) study Sites by using only odd or even bin wind speed time series. This is true when we use the moment method which consists in solving equations of the mean (Eq. 14) and standard deviation (Eq. 5).

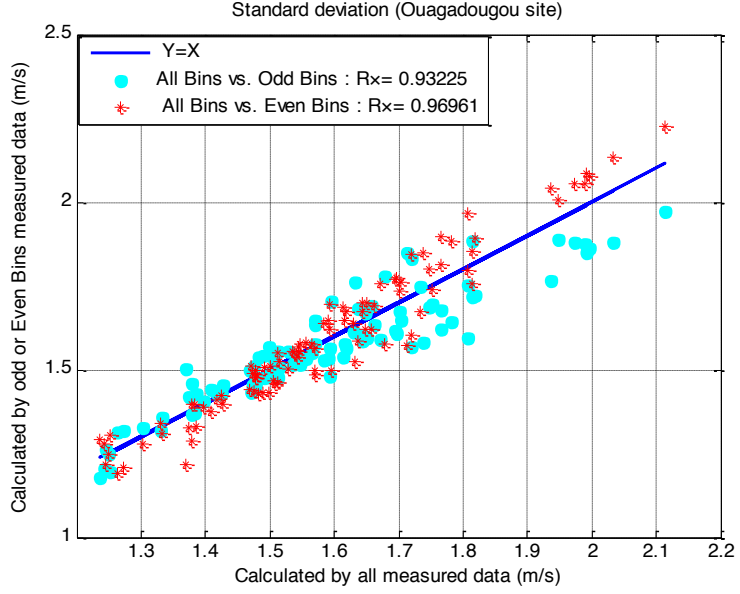

Fig. 6 Scatter plots of computed wind speed standard deviation values using all bin time series versus those computed using even bin or odd bin wind speed time series for Ouagadougou.

\subsection{Fitting histogram to Weibull functions}

Our goal in this article is to identify the most appropriate method for fitting wind speed probability distribution histograms for wind energy applications at two sites: Lomé (Togo) and Ouagadougou (Burkina Faso). From Fig. 7 and Fig. 8 it is possible to verify how the curves representing the Weibull probability density function, for each of the eighteen numerical methods considered in the analysis, match the histograms, giving an idea of which method yields the best fit to the data of wind speed collected.

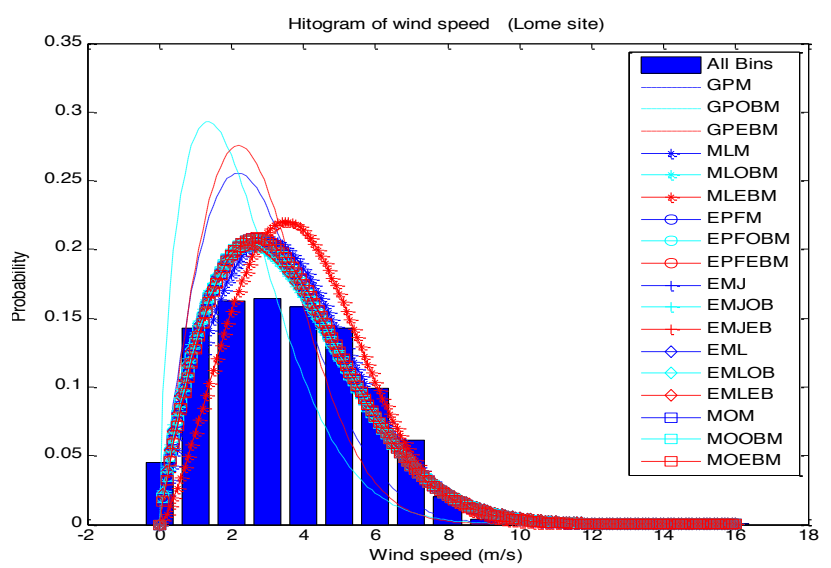

Fig. 7 Weibull distribution functions for the Lomé site (years 2004-2015).

Graphically, it is observed that, all methods present a better curve fit with the histogram of wind speed at the two sites, excepted GPM, GPOBM and GPEBM.

To choose an adequate method that better adjusts the wind speed histogram at each site, we calculated the RMSE and $\mathrm{R}^{2}$. The results in Tables 5 and 6 show that:

- at the Lomé site, only five methods (MLOBM, EMLEB, EMJEB, EML and EMJ) have RMSE lower and equal to 0.0200 and $R^{2}$ which is above 0.9665 ; the adequate method is MLOBM;

- on Ouagadougou site, only five methods (MLM, EMJOB, EMLOB, MLOBM, and EPFOBM) have 
RMSE lower and equal to 0.01477 and $\mathrm{R}^{2}$ which is above 0.9840; and the adequate method is MLM.

The results show that:

- there is a strong linear correlation between the shapes parameters $(\mathrm{K})$ estimated using all bin speed time series and the estimate using even bin or odd bin wind speed time series; unless the GPM method is used;

- there is a strong linear correlation between the scale parameters (C) estimated using all bin speed time series and the estimate using even bin or odd bin wind speed time series.

Thus we can conclude that the Weibull parameters can be estimated at the two sites using anyone of five methods (EMJ, EML, EPFM, MLM, MOM) with even or odd bin wind speed time series and then we used a linear function to estimate the Weibull parameters when the entire series of wind speed data is used

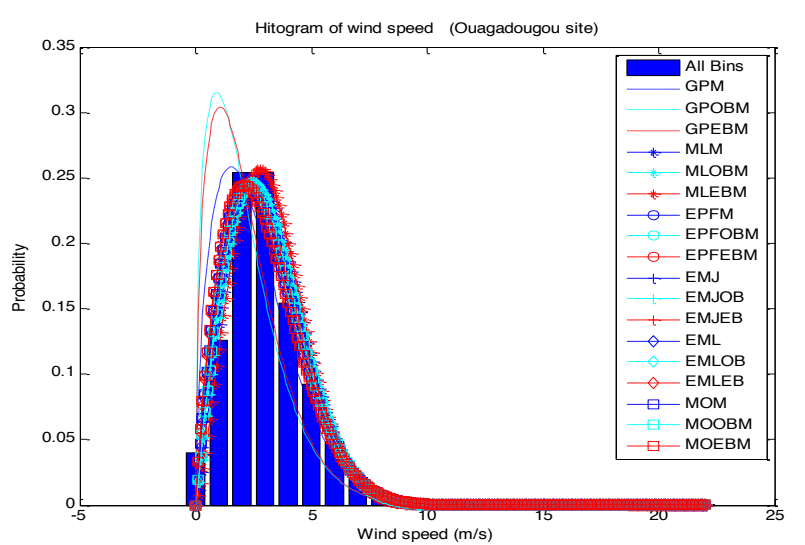

Fig. 8 Weibull distribution functions for the Ouagadougou site (years 2009-2015).

Table 5

Statistical analysis of Weibull parameters estimated for the Lomé site (years 2004-2016).

\begin{tabular}{lrrrr}
\hline Methods & $\mathbf{K}$ & $\mathbf{C}$ & $\mathbf{R M S E}$ & $\mathbf{R}^{\mathbf{2}}$ \\
\hline GPM & 1.89612 & 3.24713 & 0.04074 & 0.90607 \\
GPOBM & 1.56804 & 2.58127 & 0.05951 & 0.80298 \\
GPEBM & 1.99766 & 3.11241 & 0.04805 & 0.88165 \\
MLM & 2.03100 & 4.17884 & 0.02134 & 0.96206 \\
MLOBM & 1.79134 & 3.96250 & 0.01967 & 0.96612 \\
MLEBM & 2.38769 & 4.41967 & 0.03054 & 0.92621 \\
EPFM & 1.88456 & 3.97553 & 0.02075 & 0.96515 \\
EPFOBM & 1.86990 & 3.96502 & 0.02067 & 0.96518 \\
EPFEBM & 1.90034 & 3.98662 & 0.02085 & 0.96503 \\
EMJ & 1.82327 & 3.97037 & 0.01989 & 0.96619 \\
EMJOB & 1.81807 & 3.96046 & 0.02000 & 0.96590 \\
EMJEB & 1.82882 & 3.98088 & 0.01979 & 0.96647 \\
EML & 1.82327 & 3.97318 & 0.01985 & 0.96628 \\
EMLOB & 1.81807 & 3.96328 & 0.01995 & 0.96600 \\
EMLEB & 1.82882 & 3.98368 & 0.01974 & 0.96656 \\
MOM & 1.88656 & 3.97561 & 0.02078 & 0.96510 \\
MOOBM & 1.87190 & 3.96509 & 0.02071 & 0.96514 \\
MOEBM & 1.90234 & 3.98669 & 0.02089 & 0.96497 \\
\hline
\end{tabular}

Table 6

Statistical analysis of Weibull parameters estimated for the Ouagadougou site (years 2009-2015).

\begin{tabular}{lcccr}
\hline Methods & $\mathbf{K}$ & $\mathbf{C}$ & $\mathbf{R M S E}$ & $\mathbf{R}^{2}$ \\
\hline GPM & 1.56205 & 2.91854 & 0.03393 & 0.91999 \\
GPOBM & 1.37146 & 2.33153 & 0.05464 & 0.81172 \\
GPEBM & 1.43075 & 2.42168 & 0.05131 & 0.83444 \\
MLM & 2.10118 & 3.53456 & 0.01409 & 0.98608 \\
MLOBM & 1.97528 & 3.46123 & 0.01433 & 0.98538 \\
MLEBM & 2.24057 & 3.61116 & 0.01681 & 0.98075 \\
EPFM & 1.87026 & 3.37404 & 0.01670 & 0.98008 \\
EPFOBM & 1.94847 & 3.45396 & 0.01477 & 0.98442 \\
EPFEBM & 1.79813 & 3.29417 & 0.01949 & 0.97289 \\
EMJ & 1.89527 & 3.37560 & 0.01605 & 0.98168 \\
EMJOB & 1.98012 & 3.45524 & 0.01423 & 0.98562 \\
EMJEB & 1.81861 & 3.29592 & 0.01885 & 0.97471 \\
EML & 1.89527 & 3.37781 & 0.01604 & 0.98170 \\
EMLOB & 1.98012 & 3.45727 & 0.01424 & 0.98560 \\
EMLEB & 1.81861 & 3.29826 & 0.01883 & 0.97478 \\
MOM & 1.87126 & 3.37404 & 0.01668 & 0.98015 \\
MOOBM & 1.95747 & 3.45431 & 0.01461 & 0.98479 \\
MOEBM & 1.80013 & 3.29426 & 0.01943 & 0.97307 \\
\hline
\end{tabular}




\subsection{Comparison between the mean wind speed predicted} by the methods and the observed data

The statistical indicators introduced in Section 4 are utilized to evaluate the performances of the eighteen parameter estimation methods. Tables 7 and 8 provide the results of the performance evaluation of the eighteen methods in terms of MAPE, MABE, RMSE, RRMSE RPE, $\mathrm{R}^{2}$ and IA, respectively for the Lomé and Ouagadougou sites. It is significant to note that each statistical parameter offers different useful way of comparing the methods. Thus, the combination of all of these statistical indicators provides a possibility to compare the differences between the calculated mean wind speed from measured data and those from the Weibull distribution function within different perspectives with much higher reliability.

The results show that the accuracy of computed mean wind speed values changes when the parameter estimation method changes. It is clear that for the Lomé site when eight methods EPF, EMJ, EML, MOM, EPFEBM, EMJEB, EMLEB and MOEBM, and for the Ouagadougou site when fifteen methods of EPF, EMJ, EML, MOM, EPFEBM, EMJEB, EMLEB, MOEBM, MOOBM, EPFOBM, EMLOB, EMJOB, MLOBM, MLM and MLEBM are used to compute the Weibull parameters, the calculated mean wind speed values by the Weibull distribution function are in good agreement with the mean wind speed values computed from measured data. This conclusion is drawn because of the low values of MAPE, MABE, RMSE, RRMSE, RPE (RPE whose histograms are shown in Figs 9 and 10) and the high values of $\mathrm{R}^{2}$ and IA. On the other hand, it is found that the lowest agreements are attained when the GPOBM, GPEBM and GPM methods are applied for $\mathrm{K}$ and $\mathrm{C}$ parameter calculations

Table 7

Performance evaluation of mean wind speed calculated by all 18 methods using different statistical indicators for the Lomé site.

\begin{tabular}{lllllll}
\hline Methods & $\begin{array}{l}\text { MAPE } \\
\text { (\%) }\end{array}$ & MABE & RMSE & $\begin{array}{l}\text { RRMSE } \\
\text { (\%) }\end{array}$ & $\mathbf{R}^{2}$ & IA \\
\hline GPM & 16.555 & 0.605 & 0.623 & 17.017 & 0.978 & 0.574 \\
GPOBM & 25.454 & 0.945 & 1.021 & 27.889 & 0.788 & 0.509 \\
GPEBM & 24.655 & 0.893 & 0.910 & 24.855 & 0.968 & 0.519 \\
MLM & 8.726 & 0.271 & 0.463 & 12.659 & 0.812 & 0.734 \\
MLOBM & 7.077 & 0.219 & 0.429 & 11.733 & 0.803 & 0.786 \\
MLEBM & 11.830 & 0.377 & 0.534 & 14.588 & 0.806 & 0.637 \\
EPFM & 0.0000 & 0.000 & 0.000 & 0.000 & 1.000 & 1.000 \\
EPFOBM & 7.101 & 0.220 & 0.431 & 11.791 & 0.802 & 0.787 \\
EPFEBM & 5.197 & 0.165 & 0.289 & 7.897 & 0.957 & 0.875 \\
EMJ & 0.000 & 0.000 & 0.000 & 0.000 & 1.000 & 1.000 \\
EMJOB & 7.101 & 0.220 & 0.431 & 11.791 & 0.802 & 0.787 \\
EMJEB & 5.197 & 0.165 & 0.289 & 7.897 & 0.957 & 0.875 \\
EML & 0.053 & 0.001 & 0.001 & 0.053 & 1.000 & 0.998 \\
EMLOB & 7.108 & 0.220 & 0.432 & 11.802 & 0.802 & 0.787 \\
EMLEB & 5.211 & 0.165 & 0.290 & 7.930 & 0.956 & 0.875 \\
MOM & 0.002 & 0.000 & 0.000 & 0.003 & 1.000 & 0.999 \\
MOOBM & 7.101 & 0.220 & 0.431 & 11.791 & 0.802 & 0.787 \\
MOEBM & 5.200 & 0.165 & 0.289 & 7.903 & 0.957 & 0.875 \\
\hline
\end{tabular}

Table 8

Performance evaluation of mean wind speed calculated by all 18 methods using different statistical indicators for the Ouagadougou site

\begin{tabular}{lllllll}
\hline Methods & $\begin{array}{l}\text { MAPE } \\
(\mathbf{\% )}\end{array}$ & MABE & RMSE & $\begin{array}{l}\text { RRMSE } \\
(\mathbf{\% )}\end{array}$ & R $^{2}$ & IA \\
\hline GPM & 16.555 & 0.605 & 0.623 & 17.017 & 0.978 & 0.574 \\
GPOBM & 25.454 & 0.945 & 1.021 & 27.889 & 0.788 & 0.509 \\
GPEBM & 24.655 & 0.893 & 0.910 & 24.855 & 0.968 & 0.519 \\
MLM & 8.726 & 0.271 & 0.463 & 12.659 & 0.812 & 0.734 \\
MLOBM & 7.077 & 0.219 & 0.429 & 11.733 & 0.803 & 0.786 \\
MLEBM & 11.834 & 0.377 & 0.534 & 14.588 & 0.806 & 0.637 \\
EPFM & 0.000 & 0.000 & 0.000 & 0.000 & 1.000 & 1.000 \\
EPFOBM & 7.101 & 0.220 & 0.431 & 11.791 & 0.802 & 0.787 \\
EPFEBM & 5.197 & 0.165 & 0.289 & 7.897 & 0.957 & 0.875 \\
EMJ & 0.000 & 0.000 & 0.000 & 0.000 & 1.000 & 1.000 \\
EMJOB & 7.101 & 0.220 & 0.431 & 11.791 & 0.802 & 0.787 \\
EMJEB & 5.197 & 0.165 & 0.289 & 7.897 & 0.957 & 0.875 \\
EML & 0.053 & 0.001 & 0.001 & 0.053 & 1.000 & 0.998 \\
EMLOB & 7.108 & 0.220 & 0.432 & 11.802 & 0.802 & 0.787 \\
EMLEB & 5.211 & 0.165 & 0.290 & 7.930 & 0.956 & 0.875 \\
MOM & 0.002 & 0.000 & 0.000 & 0.003 & 1.000 & 0.999 \\
MOOBM & 7.101 & 0.220 & 0.431 & 11.791 & 0.802 & 0.787 \\
MOEBM & 5.200 & 0.165 & 0.289 & 7.903 & 0.957 & 0.875 \\
\hline
\end{tabular}




\subsection{Comparison between wind speed standard deviation predicted by the methods and measured data}

The same statistical analyses to compare the mean wind speed predicted by the methods to that calculated from collected data are made here.

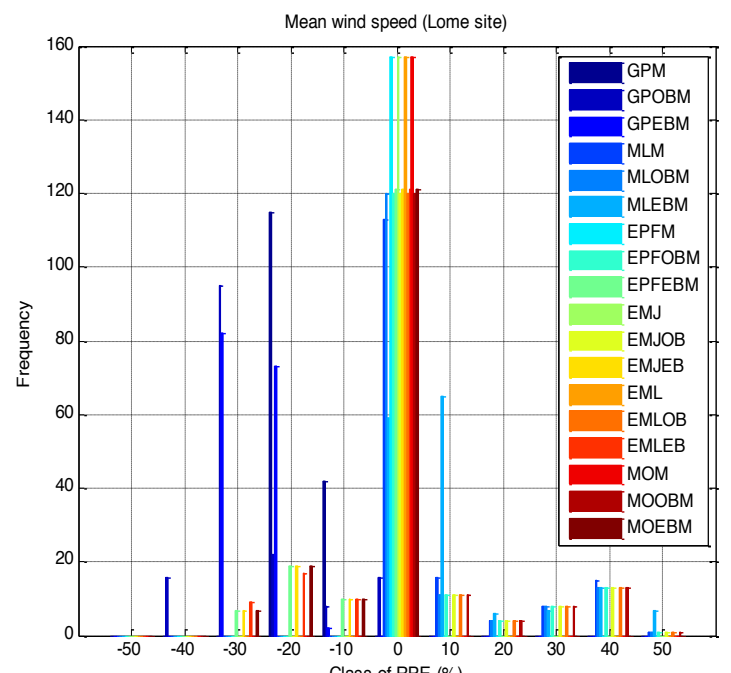

Fig. 9 Histograms of RPE (\%) of mean wind speed predicted with 18 methods for Lomé.

Tables 9 and 10 provide the results of the performance evaluation for the eighteen methods in terms of MAPE, MABE, RMSE, RRMSE, $\mathrm{R}^{2}$ and IA, respectively for the Lomé and Ouagadougou sites. The results show that the accuracy of computed wind speed standard deviation values changes when the parameter estimation method changes. It is clear that for the Lomé site when four methods namely EML, EMJ, EMLEB and EMJEB, and for the Ouagadougou site when six methods namely EML, EMJ, MOM, EPF, EMJEB, EMLEB and MOEBM are used to compute the Weibull parameters, the calculated wind speed standard deviation by the Weibull distribution function are in good agreement with the wind speed standard deviation values computed from measured data. This conclusion is drawn because of the low values of MAPE, MABE, RMSE, RRMSE, RPE (RPE whose histograms are shown in Figs 11 and 12). On the other hand, it is found that the lowest agreements are obtained when the GPOBM, GPEBM and GPM methods are applied for $\mathrm{K}$ and $\mathrm{C}$ parameter calculation.

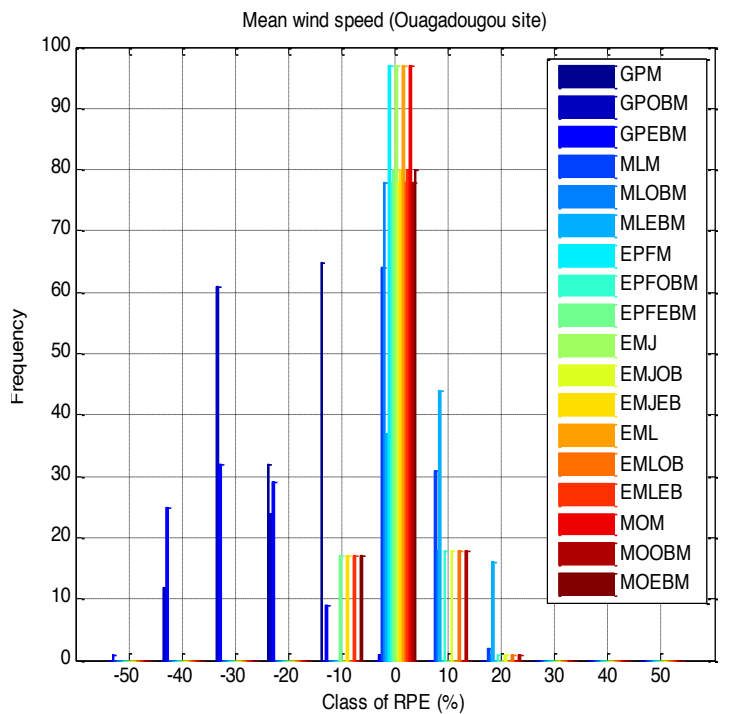

Fig. 10 Histograms of RPE (\%) of mean wind speed predicted with 18 methods for Ouagadougou.

Table 9

Performance evaluation of the standard deviation of wind speed calculated by all 18 methods using different statistical indicators for the Lomé site

\begin{tabular}{lcccccc}
\hline Methods & MAPE(\%) & MABE & RMSE & RRMSE(\%) & $\mathbf{R}^{\mathbf{2}}$ & IA \\
\hline GPM & 19.407 & 0.397 & 0.490 & 24.424 & 0.238 & 0.500 \\
GPOBM & 19.059 & 0.387 & 0.514 & 25.637 & 0.234 & 0.516 \\
GPEBM & 26.328 & 0.535 & 0.628 & 31.309 & 0.186 & 0.498 \\
MLM & 8.175 & 0.176 & 0.272 & 13.601 & 0.309 & 0.534 \\
MLOBM & 6.601 & 0.143 & 0.237 & 11.839 & 0.234 & 0.500 \\
MLEBM & 13.863 & 0.287 & 0.358 & 17.889 & 0.327 & 0.495 \\
EPFM & 4.260 & 0.089 & 0.111 & 5.552 & 0.944 & 0.666 \\
EPFOBM & 6.632 & 0.145 & 0.242 & 12.099 & 0.284 & 0.515 \\
EPFEBM & 6.256 & 0.129 & 0.172 & 8.605 & 0.760 & 0.576 \\
EMJ & 0.994 & 0.020 & 0.021 & 1.059 & 0.999 & 0.932 \\
EMJOB & 6.177 & 0.134 & 0.234 & 11.707 & 0.257 & 0.518 \\
EMJEB & 3.606 & 0.071 & 0.084 & 4.204 & 0.957 & 0.810 \\
EML & 0.942 & 0.019 & 0.020 & 1.002 & 0.999 & 0.935 \\
EMLOB & 6.189 & 0.134 & 0.234 & 11.701 & 0.256 & 0.517 \\
EMLEB & 3.564 & 0.070 & 0.083 & 4.173 & 0.957 & 0.812 \\
MOM & 4.154 & 0.087 & 0.112 & 5.591 & 0.956 & 0.683 \\
MOOBM & 6.661 & 0.145 & 0.244 & 12.169 & 0.306 & 0.531 \\
MOEBM & 6.225 & 0.128 & 0.173 & 8.666 & 0.771 & 0.588 \\
\hline
\end{tabular}


Table 10

Performance evaluation of the standard deviation of wind speed calculated by all 18 methods using different statistical indicators for the Ouagadougou site.

\begin{tabular}{lllllll} 
Methods & MAPE (\%) & MABE & RMSE & RRMSE (\%) & $\mathbf{R}^{2}$ & IA \\
\hline GPM & 12.385 & 0.203 & 0.281 & 17.765 & 0.326 & 0.513 \\
GPOBM & 15.291 & 0.247 & 0.319 & 20.144 & 0.469 & 0.539 \\
GPEBM & 18.570 & 0.299 & 0.370 & 23.350 & 0.421 & 0.530 \\
MLM & 5.679 & 0.093 & 0.110 & 6.987 & 0.959 & 0.673 \\
MLOBM & 3.481 & 0.058 & 0.077 & 4.916 & 0.935 & 0.770 \\
MLEBM & 10.464 & 0.167 & 0.189 & 11.986 & 0.881 & 0.590 \\
EPFM & 1.929 & 0.030 & 0.036 & 2.334 & 0.985 & 0.887 \\
EPFOBM & 4.743 & 0.075 & 0.099 & 6.281 & 0.855 & 0.684 \\
EPFEBM & 3.111 & 0.048 & 0.063 & 3.983 & 0.951 & 0.848 \\
EMJ & 1.010 & 0.016 & 0.017 & 1.090 & 0.999 & 0.945 \\
EMJOB & 3.670 & 0.061 & 0.079 & 5.004 & 0.933 & 0.763 \\
EMJEB & 3.028 & 0.048 & 0.060 & 3.832 & 0.969 & 0.864 \\
EML & 0.955 & 0.015 & 0.016 & 1.029 & 0.999 & 0.948 \\
EMLOB & 3.658 & 0.061 & 0.078 & 4.980 & 0.933 & 0.764 \\
EMLEB & 3.027 & 0.048 & 0.060 & 3.832 & 0.969 & 0.864 \\
MOM & 0.769 & 0.013 & 0.025 & 1.577 & 0.995 & 0.952 \\
MOOBM & 3.588 & 0.059 & 0.078 & 4.967 & 0.927 & 0.765 \\
MOEBM & 2.928 & 0.046 & 0.060 & 3.825 & 0.960 & 0.860 \\
\hline
\end{tabular}

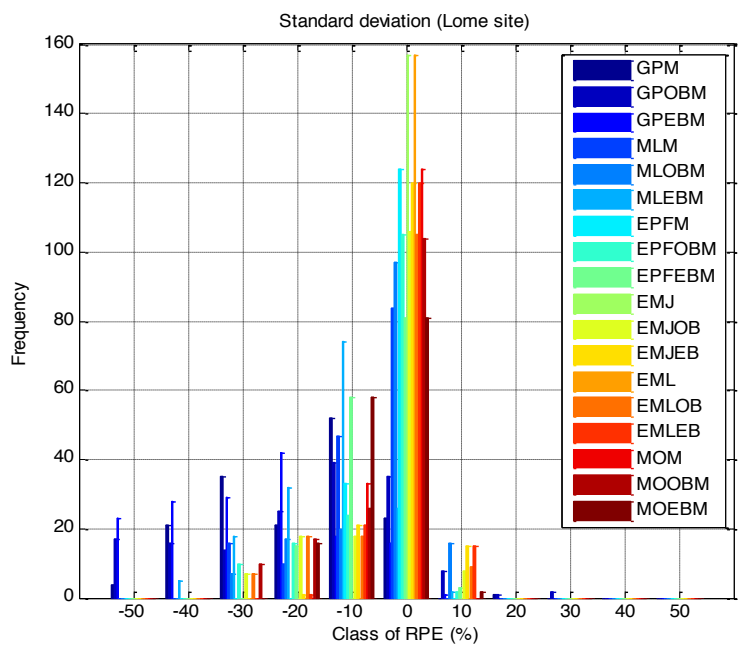

Fig. 11 Histograms of RPE (\%) for wind speed standard deviations predicted with 18 methods for Lomé.

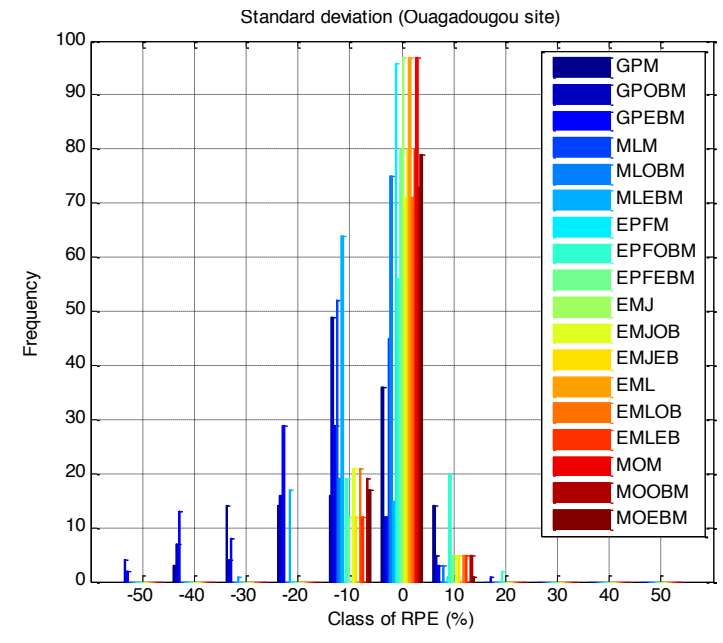

Fig. 12 Histograms of RPE (\%) for wind speed standard deviations predicted with 18 methods for Ouagadougou.

\section{Conclusion}

The following conclusions can be drawn from the preceding analysis:

- the approaches based on odd or even class wind speed time series with $1 \mathrm{~m} / \mathrm{s}$ as bin size using Maximum Likelihood Method (MLM), the Energy Pattern Factor Method (EPFM), the Empirical Method of Justus (EMJ), the Empirical Method of Lysen (EML) and the Moment method (MOM) are efficient methods for determining the $\mathrm{K}$ and $\mathrm{C}$ parameters to fit Weibull distribution curves to wind speed data collected in Lomé, Togo and Ouagadougou, Burkina Faso analyzed using statistical tests;

- the maximum likelihood with the odd bin time series method (MLOBM) is an adequate method that gave lower RMSE value of Weibull parameters $\mathrm{K}=1.79134$ and $\mathrm{C}=3.96250 \mathrm{~m} / \mathrm{s}$ at the Lomé site using the whole data collected; 
- the MLM is an adequate method that yields lower RMSE value of the Weibull parameters $\mathrm{K}=2.10118$ and $\mathrm{C}=3.53456 \mathrm{~m} / \mathrm{s}$ at the Ouagadougou site using the whole data collected;

- the accuracy of computed mean and standard deviation of wind speed values changes when the parameter estimation method changes at the Lomé and Ouagadougou sites;

- in the evaluation of the mean and standard deviation, the GPM, the Graphical with Even Bin time series Method (GPEBM) and the Graphical with Odd Bin time series Method (GPOBM) are the least effective methods for fitting Weibull distribution curves to the wind speed using the data analyzed for the Lomé and Ouagadougou sites;

- there is a linear relationship between the Weibull parameters K and C estimated by MLM, EPFM, EMJ, EML and MOM using odd or even class wind speed time series and all class wind speed time series; this result empowers one to compress wind data by removing either even or odd bins (depending on the site) prior to any available energy assessment while still expecting the same outcome as when the entire data is used.

\section{References}

Ahmed, S.A., (2013). Comparative study of four methods for estimating Weibull parameters for Halabja, Iraq. International Journal of Physical Sciences, 8(5), 186-192..

Al-Mulali, U., \& Sab, C. N. B. C., (2012). The impact of energy consumption and $\mathrm{CO} 2$ emission on the economic growth and financial development in the Sub Saharan African countries. Energy,, 180-186.

Bugaje I. M., (2006). Renewable energy for sustainable development in Africa: a review,. Renew. Sustain. Energy Rev.,603-612.

Celik, A.N., (2004). A statistical analysis of wind power density based on the Weibull and Rayleigh models at the southern region of Turkey. Renewable Energy, 29(4), 593-604.

Dahmouni, A. W., Salah, M. B., Askri, F., Kerkeni, C., \& Nasrallah, S. B., (2011). Assessment of wind energy potential and optimal electricity generation in Borj-Cedria , Tunisia. Renewable and Sustainable Energy Reviews, 15(1), 815-820.

Dinler, A. \& Akdag, S.A., (2009). A new method to estimate Weibull parameters for wind energy applications. 50, 1761-1766.
Garcia, A., Torres, J. L., Prieto, E., \& De Francisco, A., (1998). Fitting wind speed distributions: a case study. Solar Energy, 62(2), 139-144..

Kasra, M., Omid,A., Ali, M. \& Navid, G.M.J., (2016). Assessing different parameters estimation methods of Weibull distribution to compute wind power density. Energy Conversion and Management, 108(November), 322-335.

Kidmo, D. K., Danwe, R., Doka, S. Y., \& Djongyang, N., (2015). Statistical analysis of wind speed distribution based on six Weibull Methods for wind power evaluation in Garoua, Cameroon.,18,105-125..

Kimatu, W., Ayenagbo, J. N., Rongcheng, K., (2011). A model for a sustainable energy supply strategy for the socialeconomic development of Togo.

Legates, D. R., \& McCabe, G. J., (1999). Goodness-of-fit measures in hydrologic and hydro climatic model validation,. Water Resour. Res.,, 35, 233-241.

Mostafaeipour, A., Sedaghat, A., Dehghan-Niri, A. A., \& Kalantar, V., (2011). Author's personal copy Wind energy feasibility study for city of Shahrbabak in Iran. Renewable and Sustainable Energy Reviews, 15, 25452556.

Rocha, P. A. C., de Sousa, R. C., de Andrade, C. F., \& da Silva, M. E. V., (2012). Comparison of seven numerical methods for determining Weibull parameters for wind energy generation in the northeast region of Brazil. Applied Energy, 89(1), 395-400.

Sahin, A.D., (2004). Progress and recent trends in wind energy. Progress in Energy and Combustion Science, 30(5), 501543.

Salami, A.A., Ajavon, A. S. A, Kodjo, M.K. \& Bedja, K., (2013). Contribution to improving the modeling of wind and evaluation of the wind potential of the site of Lome: Problems of taking into account the frequency of calm winds. Renewable Energy, 50, 449-455. A

Salami, A.A., Ajavon, A. S. A, Kodjo, M.K. \& Bedja, K., (2016). Evaluation of Wind Potential for an Optimum Choice of Wind Turbine Generator on the Sites of Lome, Accra, and Cotonou Located in the Gulf of Guinea.,. Int. Journal of Renewable EnergyDevelopment,, 5(3), 211-223. Seguro, J. V \& Lambert, T.W., (2000). Modern estimation of the parameters of the Weibull wind speed distribution for wind energy analysis. Journal of Wind Engineering and Industrial Aerodynamics, 85(1), 75-84.

Yuan F. Q. Barabadi A., L.J.M.G.A.H.S., (2015). Performance evaluation for maximum likelihood and moment parameter estimation methods on classical two Weibull distributions. Ind. Eng. Eng. Manag. (IEEM), 2015 IEEE Int. Conf.,, 2015, 802-806. 